\title{
Rare genetic variants in cellular transporters, metabolic enzymes, and nuclear receptors can be important determinants of interindividual differences in drug response
}

\author{
Mikael Kozyra, Medical student ${ }^{1}$, Magnus Ingelman-Sundberg, PhD ${ }^{1}$ and Volker M. Lauschke, PhD $^{1}$
}

\begin{abstract}
Purpose: In this study we characterized the genetic variability of 146 clinically relevant genes influencing drug pharmacokinetics in African and European subpopulations, which are key determinants for interindividual variations in drug efficacy and adverse drug reactions.

Methods: By integrating data from the 1000 Genomes Project $(n=$ 1,092 individuals) and the Exome Sequencing Project (ESP; $n=6,503$ individuals), single-nucleotide variants (SNVs) were identified and analyzed regarding frequency, functional consequences, and ethnic diversity.

Results: In total, we found 12,152 SNVs in exons, 312 of which were novel. The majority of variants were rare (minor allele frequency (MAF) $<1 \%$; 92.9\%) and nonsynonymous (56.2\%). We calculated that individuals of European and African descent harbor, on average, 100.8 and 121.4 variants across the 146 pharmacogenes studied,
\end{abstract}

respectively. Additionally, by analyzing variation patterns across these populations, we pinpointed potential priority genes for population-adjusted genetic profiling strategies. Furthermore, we estimated, based on our variant frequency analyses, that approximately $30-40 \%$ of functional variability in pharmacogenes can be attributed to rare variants.

Conclusions: Our results indicate that these clinically important genes are genetically highly variable and differ considerably between populations. Furthermore, the large extent of rare variants emphasizes the need for sequencing-based approaches and effective functionality predictions to allow for true personalized medicine.

Genet Med advance online publication 21 April 2016

Key Words: drug development; genetic variation; personalized medicine; pharmacogenes; rare variants

\section{INTRODUCTION}

The development of next-generation sequencing platforms and the mapping of the human genome have transformed the field of pharmacogenetics into pharmacogenomics, thus expanding its scope from a candidate-driven approach to genome-wide studies of associations. The vast amount of genetic information opens new possibilities to describe and categorize the extent of genetic polymorphisms in the human genome at the population level.

Interindividual differences in drug pharmacokinetics (PK) and pharmacodynamics (PD) can cause the loss of treatment efficacy or give rise to adverse drug reactions (ADRs) with negative consequences for patients. In addition, ADRs can be as costly for society when they occur with the initial treatment for symptoms. ${ }^{1}$ They are the prime cause of drug withdrawal from the market, resulting in significant costs for the pharmaceutical industry that have been calculated as approximately US $\$ 2.6$ billion per new drug. ${ }^{2}$ In recent decades, much research has aimed to identify the genetic basis for interindividual differences in drug response and toxicity; in particular, this work has resulted in a series of genomic drug labels by the Food and Drug Administration (FDA) ${ }^{3}$ (http://www.fda.gov/drugs/scienceresearch/researchareas/pharmacogenetics/ucm083378.htm) and the European Medicines Agency ${ }^{4}$ to optimize drug treatment. These studies also uncovered a vast repertoire of genetic variants with profound impact on drug metabolism, some of which have been successfully translated in clinical practice. ${ }^{5}$

Much research has been focused on the cytochrome P450 $(C Y P)$ superfamily of genes because these are major contributors to drug metabolism, primarily in the liver. The impact of this variation on drug response and ADRs is extensive; recently, a comprehensive overview of the genetic variability within the CYP super gene family with important implications for personalized medicine was published. ${ }^{6}$ Yet, a plethora of other genes such as other phase I enzymes, phase II conjugation enzymes, transporters, cytochrome reductases, and nuclear receptors, hereafter jointly termed "pharmacogenes," also impact PK and PD directly or indirectly and can cause ADRs. ${ }^{7}$

Genes encoding transporter proteins belong primarily to two large gene families: the ATP-binding cassette $(A B C)$ superfamily, whose members export substances out of cells, and the solute carrier $(S L C)$ superfamily, which mostly mediate molecule influx. They can translocate a vast variety of compounds like xenobiotics and drugs, thereby impacting $\mathrm{PD}$ and PK. ${ }^{8}$ Transporters of the $\mathrm{ABC}$ family are important in cancer therapy because they can confer resistance to chemotherapy by 
extruding chemotherapeutic drugs; thus, they are associated with poor prognosis. ${ }^{9}$

The SLC transporter family contains more than 300 genes with selectivity to structurally diverse compounds whose variants impact drug PK. One such example is given by genetic polymorphisms in SLCO1B1, a gene encoding the cellular transporter OATP1B1 that mediates influx of a wide variety of pharmaceutical substances like various statins, bosentan, and troglitazone..$^{10}$ The single-nucleotide variant (SNV) rs4149056 in SLCO1B1 (SLCO1B1 ${ }^{* 5}$ ) has been shown to profoundly influence statin uptake, resulting in lower inhibition of HMG-CoA and increased blood cholesterol levels. ${ }^{11}$ Furthermore, the rs4363657 variant, which is in almost complete linkage disequilibrium with rs4149056, showed a strong association with statin-induced myopathy. ${ }^{12}$ Inversely, treatment efficacy of the antidiabetes drug repaglinide is increased in variant carriers because hepatic uptake primes the compound for biotransformation into inactive metabolites. ${ }^{13}$

In addition to transporters, phase II enzymes constitute an important class of pharmacogenes that are responsible for the detoxification and inactivation of drugs, mostly by transfer of chemical moieties that render the drug, toxin, or hormone more hydrophilic and facilitate excretion. Major phase II pathways include conjugation with glucuronides by UDPglucuronosyltransferases (UGTs), the addition of glutathione by glutathione-S-transferases (GSTs), and sulfonation catalyzed by sulfotransferases (SULTs). UGTs contribute to the biotransformation of drugs and other exogenous substances, such as tobacco smoke carcinogens as well as environmental and dietary pollutants. ${ }^{14}$ Kinetics of these detoxification processes influence exposure to toxic or carcinogenic substances and thus can modify the susceptibility to exposure-induced diseases such as cancer. ${ }^{14}$ Furthermore, genetic variants can influence the susceptibility to drug-induced toxicity, as exemplified by ADRs seen upon treatment with irinotecan..$^{15}$ GSTs catalyze the transfer of reduced glutathione to unipolar compounds such as halogenonitrobenzenes, quinones, and unsaturated carbonyls. ${ }^{16}$ Genetic variants in GSTs affect the metabolism of busulfan and cyclophosphamide, two compounds with narrow therapeutic ranges used in conditioning prior to hematopoietic stem cell transplantations. ${ }^{17}$ Sulfonation of drugs, environmental chemicals, and natural products via SULTs can result in detoxification or metabolic activation to mutagenic electrophiles. ${ }^{18}$ In total, approximately $100 \mathrm{FDA}$-approved drugs are sulfated by SULTs, and variants have been shown to modulate drug sulfation. ${ }^{19,20}$

Nuclear receptors such as the aryl hydrocarbon receptor, constitutive androstane receptor, and pregnane $\mathrm{X}$ receptor can be important indirect determinants of PK and PD because they are key regulators mediating transcriptional responses to environmental stimuli and control expression of a variety of phase I and phase II enzymes and transporters. ${ }^{21}$

Although approximately $35 \%$ of drugs with pharmacogenomic labels listed by the FDA are related to variants in the pharmacogenes analyzed in this article (http://www.fda.gov/ drugs/scienceresearch/researchareas/pharmacogenetics/ ucm083378.htm), a comprehensive overview of the inventory of genetic variation on a population level is still lacking. Here, we present an extensive data set of human polymorphisms within important pharmacogenes based on two population sequencing projects: the ESP of the National Heart, Lung, and Blood Institute ${ }^{22}$ and the 1000 Genomes Project. ${ }^{23}$ We provide an overview and classification of variants across populations and assess the contribution of rare variants to overall functional variability. Furthermore, the data presented here deliver new insights into genetic variants that potentially affect drug efficacy and can thus incentivize the adoption of this knowledge into clinical practice.

\section{MATERIAL AND METHODS}

\section{Data collection and filtration}

A list of important pharmacogenes was collected from http:// www.pharmaadme.org and expanded with genes from the literature that could play a role in the PK or PD. Data on each of the 146 genes were collected from ESP database (http://www.nhlbi. nih.gov/) release ESP6500SI-V222 and from 1000 Genomes Project release 20130502 (http://www.ncbi.nlm.nih.gov/variation/tools/1000genomes). ${ }^{23}$ Information regarding variants was retrieved from NCBI dbSNP release 134 (http://www.nlm.nih. gov/SNP/), Ensembl variation (http://www.ensembl.org/info/ genome/variation/index.html), and UCSC genome browser (http://genome.ucsc.edu/). Variation rates were calculated as follows:

$$
\mathrm{VR}=\frac{\operatorname{SUM}\left(\mathrm{MAF}_{\mathrm{EA}} \times n_{\mathrm{EA}}\right)+\operatorname{SUM}\left(\mathrm{MAF}_{\mathrm{AA}} \times n_{\mathrm{AA}}\right)}{\left(n_{\mathrm{EA}}+n_{\mathrm{AA}}\right) \times 100}
$$

where $\mathrm{SUM}(\mathrm{MAF})$ is the aggregated minor allele frequencies and $n$ is the allele sample size for European Americans or African Americans, respectively. All chromosome files were obtained from http://ftp.1000genomes.ebi.ac.uk/vol1/ $\mathrm{ftp} /$ phase1/analysis_results/supporting/accessible_genome_ masks/ and analyzed using UGENE (http://ugene.unipro.ru). Gene coordinates were obtained from GeneCards (version 3.12.396 26 May 2015).

\section{Quality assessment of sequencing methods}

The suitability of short-read sequencing technologies was assessed as described by Drögemöller et al. ${ }^{24}$ GC contents and gene paralogues were identified using Ensembl BioMart (http://www.ensembl.org/biomart/martview/ 3f677381fladc3869a4976e7dfc4de22). The inaccessible genomic fraction that is not reliably readable by short-read sequencing technologies was calculated using data provided by the 1000 Genomes Project ("strict mask") in Python.

\section{Computational predictions of functional effects of nonsynonymous variants}

Functionality of variants was assessed as in the work by Fujikura et al. ${ }^{6}$ and references therein. Additionally, SNPs $3 \mathrm{D}^{25}$ was used to incorporate structural considerations into the functionality assessments. SNPs 3D scores less than 0 were 
considered deleterious following cutoff values from the respective publication.

\section{Statistical analyses}

Statistical analyses were performed using Excel (Microsoft) and Prism 6 (GraphPad software). For comparisons between two groups, heteroscedastic two-tailed Student's $t$-tests were used. $P$ values less than 0.05 were considered statistically significant. Significant differences between standard deviations were calculated using $F$-tests.

\section{RESULTS}

The majority of variants in pharmacogenes are very rare We chose pharmacogenes on the basis of a consensus list defined by academia and industry representatives and expanded it with further genes of interest implicated in drug PK or PD. As a result, 146 genes were analyzed by integrating data from the 1000 Genomes Project and the ESP of the National Heart, Lung, and Blood Institute (Figure 1a). By analyzing data from more than 6,500 individuals from two ethnic groups, we detected a total of 19,328 SNVs, 12,152 (62.9\%) of which were located in exons (see Supplementary Table S1 online for a complete list of all found exonic variants). Variants were distributed over all genes analyzed with the exception of SULT1A3. SULT1A3 is $99.8 \%$ identical to SULT1A4 and shares part of its coding sequence with the $S L X 1 B$ gene, a highly conserved endonuclease involved in the replication of ribosomal DNA clusters, ${ }^{26}$ which could at least partially explain the lack of detected variants.

We found 6,225 and 6,258 variants in $A B C$ (22 genes) and $S L C$ transporter genes (49), respectively. Furthermore, we identified 1,783 variants in phase I enzymes (14) and 2,528 SNVs in phase II enzymes, of which 1,219, 793, 263, and 253 variants were allotted to UGTs (16), GTSs (14), SULTs (4), and others (5), respectively (see Supplementary Table S2 online). Among the nuclear receptors in our pharmacogenetic panel (15 genes) we found 1,343 variants, and another 1,191 were detected in pharmacogenes with miscellaneous functions ( 7 genes, Figure 1b). Because intronic variants are only byproducts in the ESP that are not systematically covered, we focused our further analyses exclusively on exonic variants.

From these, most variants per gene were detected in $A B C$ transporters (167.3), whereas GSTs showed least variants (32.8). To directly compare the variability between gene families, we normalized the number of SNVs to the length of the corresponding gene and identified 28.2 \pm 12.1 SD SNVs per kilobase pair (kbp) of transcript overall. UGT enzymes (95\% CI: 32-38.7 SNVs/kbp), and ABC transporters (95\% CI: 26.4-36.2 SNVs/kbp) showed the highest variability, whereas nuclear receptors were most conserved (95\% CI: 7.6-14.4 SNVs/kbp; Figure 1c). It should be noted, however, that the genomic architecture of UGTs might contribute to the consistently high variability because the UGT1A subfamily consists of nine isoforms derived from a single locus that only differs in the first exon. ${ }^{27}$ To put these values into perspective, we calculated the genetic variability of 13 CYP genes (CYP1A1, CYP1A2, CYP2A6, CYP2B6, CYP2C8, CYP2C9, CYP2C19, CYP2D6, CYP2E1, CYP2J2, CYP3A4, CYP3A5, CYP4F2), hereafter termed CYP13 , that account for the majority of all phase I drug reactions in humans. The variability of CYP-13 (95\% CI: 27.1-45.5 SNVs/ $\mathrm{kbp}$ ) was similar to that of genes in the UGT family, indicating that some pharmacogenetic gene groups are as variable as the highly diverse $C Y P$ gene family.

We detected 312 novel exonic variants with numbers of SNVs ranging from 0 to 10 per individual gene. Overall, most novel SNVs were found in SLC (122 novel variants) and $A B C$ transporters (62; Figure 1d). Considering individual genes, $A B C C 12$ (10 novel variants), SLCO1B3 (10), and ABCC2 (8) showed the highest numbers of novel SNVs, whereas 29 genes, including VKORC1, CYBR1, PPARA, and HNF4A, harbored none (see Supplementary Table S2 online). The majority of these novel variants $(70.5 \%)$ were frameshift variants; the rest were classified as coding (16.7\%), UTR (9.9\%), or splice-site mutations (3.2\%).

Importantly, most SNVs we found were either rare $(\mathrm{MAF}<1 \%$; $92.9 \%)$ or very rare (MAF $<0.1 \% ; 82.7 \%)$, reflecting recent explosive population growth ${ }^{28}$ (Figure 1e). Most very rare variants were found in UGTs and $A B C$ transporters, thus suggesting increased evolutionary plasticity in these gene classes (Figure 1f).

Previous studies showed that the quality of short-read sequencing projects can significantly vary between loci and are influenced by, for example, repetitive sequences, high similarity to paralogous genes, and high GC contents, which in turn can impact the fidelity of variant calls. ${ }^{24}$ To systematically analyze the complexity of all pharmacogenes, we calculated the fraction of sequence that is classified as inaccessible by short-read sequencing technologies. Notably, the fraction of inaccessible genome was highest in SULT genes, with an average of 73\% of genomic intervals deemed inaccessible due to substantial sequence identity. Although we detected a statistically significant correlation between the number of SNVs and the percentage of inaccessible genomes, this correlation was weak and could only explain $3.3 \%$ of the differences in variants between genes $\left(R^{2}=0.033, P=0.03\right)$. Importantly, no correlation was detected between the number of novel variants and the inaccessible genome fraction $\left(R^{2}=0.003, P=0.53\right)$, suggesting that novel variants are not calling artifacts.

\section{The majority of SNVs were nonsynonymous}

The largest fraction of SNVs in pharmacogenes caused amino acid changes ( $n=6,827,56.2 \%)$, whereas $4,047(33.3 \%)$ were synonymous (Figure 2a). Interestingly, although the extent of missense variants was relatively similar across all pharmacogenes we studied here $(55.4 \pm 9 \% \mathrm{SD})$, this fraction was similar in magnitude but significantly more variable in the CYP supergene family $(45.9 \pm 33 \% \mathrm{SD}$; data derived from ref.6; difference between standard deviations: $P<0.0001)$.

When only missense variants were considered, $A B C A 4$ (230) and $A B C C 3$ (146) showed the highest numbers of overall variants (Figure 2b). ABCA4 is a rod cell-specific photoreceptor 
a

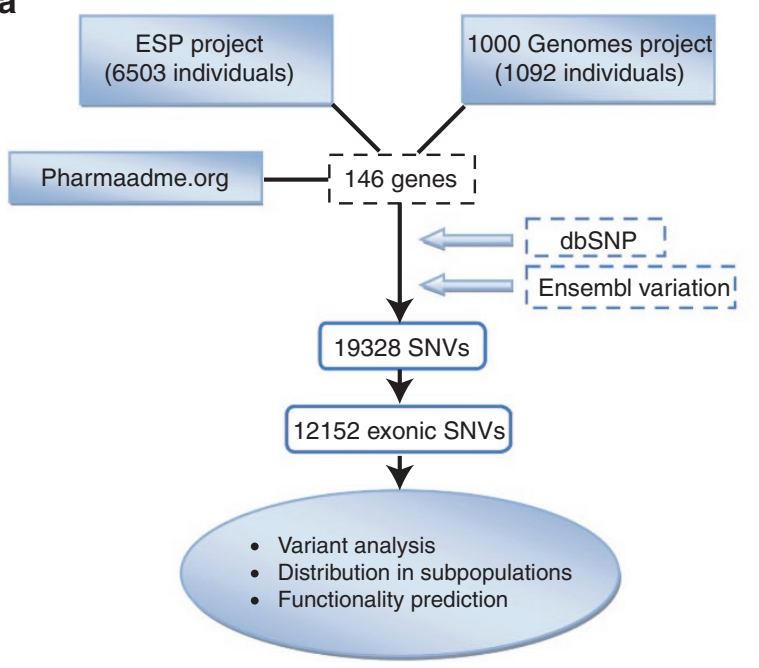

C

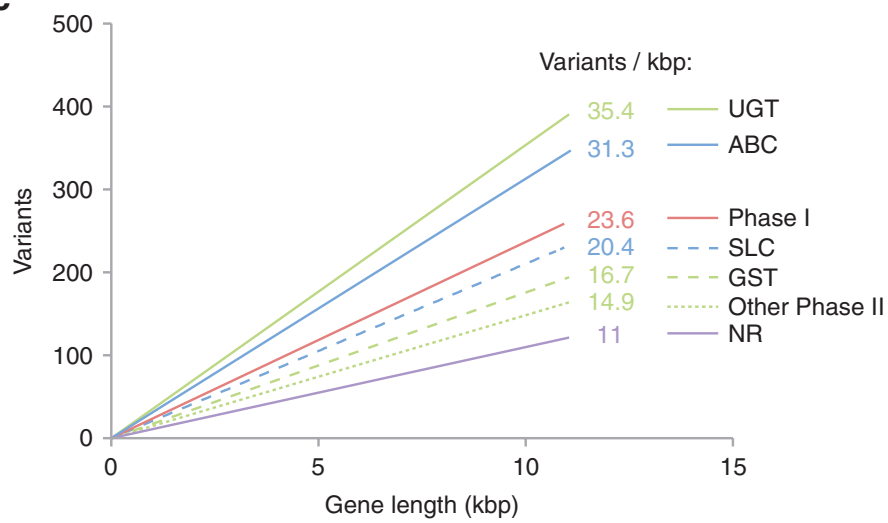

e

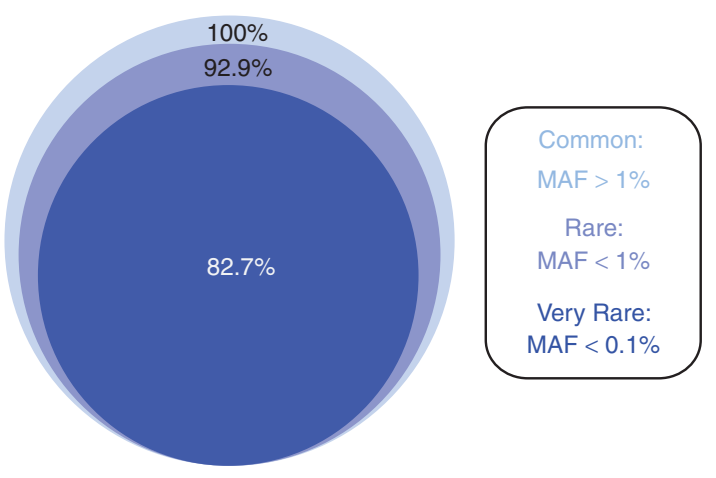

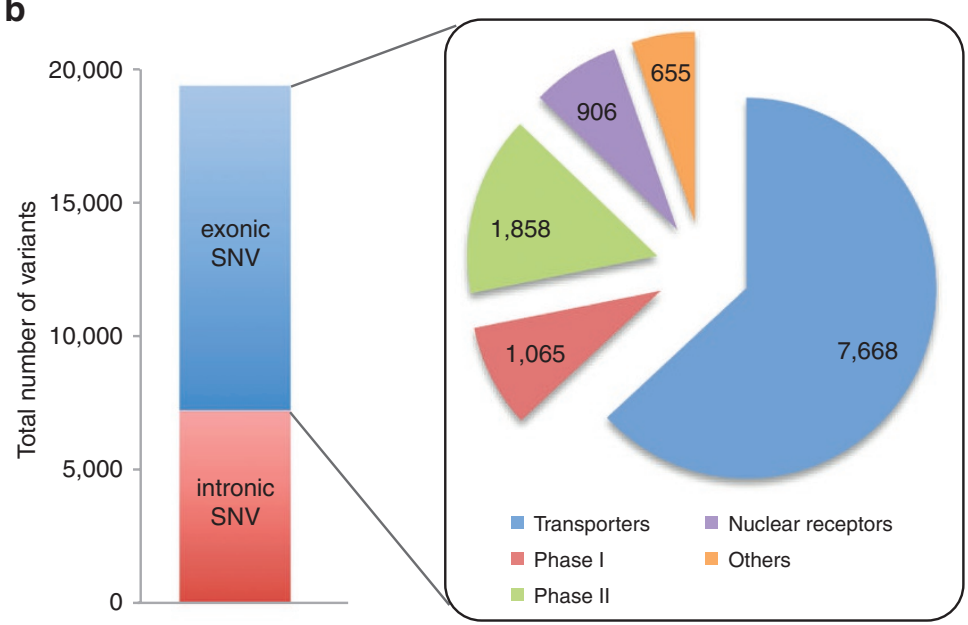

d

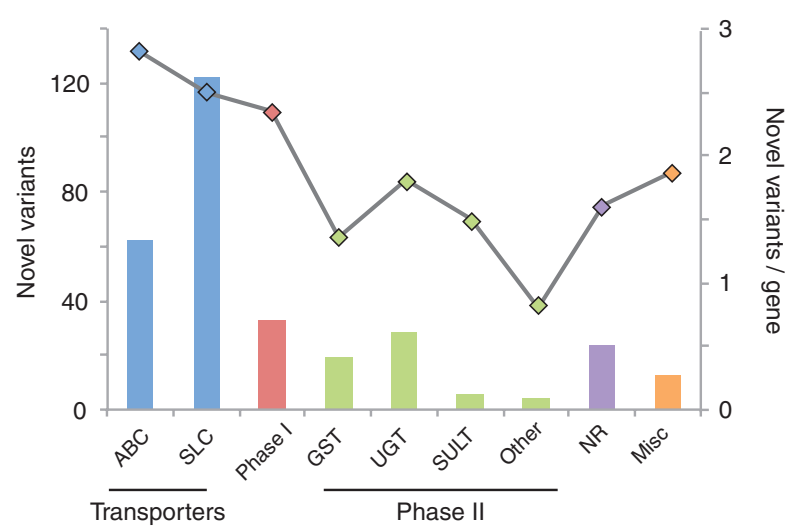

f

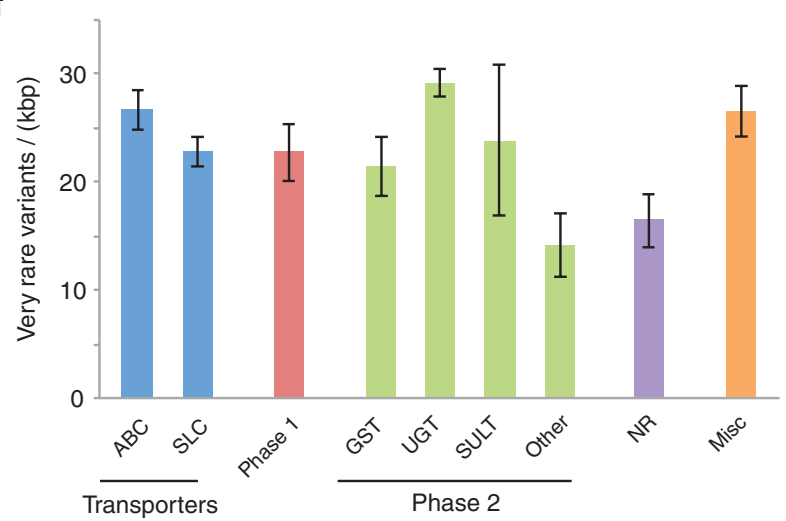

Figure 1 The vast majority of variants detected in a panel of 146 pharmacogenes were rare. (a) Schematic depicting the workflow for the identification of sequence variants. Genes of interest were selected based on a consensus list of drug-metabolizing genetic biomarkers with relevance for clinical trials and US Food and Drug Administration drug submissions. This list was complemented with additional genes with importance for pharmacokinetics. Integration of variant information data from the 1000 Genomes Project and the Exome Sequencing Project resulted in the identification of 19,328 SNVs, 62.9\% $(n=12,152)$ of which were located in exons. (b) Stacked column and pie chart showing the variant composition of all pharmacogenes. Of the 12,152 exonic variants identified, most variants were located in transporters $(7,668$ SNVs) and genes encoding phase II enzymes $(1,858)$. (c) Regression model showing correlations of gene length and number of variants organized by gene classes. Transporters, genes encoding phase I and II enzymes, and nuclear receptors are colored blue, red, green, and purple, respectively. Note that genes encoding UGT enzymes (35.4 variants/kbp) and ABC transporters (31.3 variants/kbp) showed the highest numbers of variants, whereas nuclear receptors were most conserved. (d) The number of novel variants is shown for each gene class with columns. Most novel variants were detected in SLC transporter genes; however, when normalizing for number of genes in each gene family, most variants are detected in $A B C$ transporters, whereas phase II enzymes harbor the overall least novel variants (overlaid gray line). (e) The majority of variants found in pharmacogene exomes were rare $(92.9 \%)$ or very rare $(82.7 \%)$, with MAFs $<1 \%$ or $0.1 \%$, respectively. (f) When normalizing for gene length, most very rare variants were identified in UGT (29.18 variants/kbp $\pm 1.3 \mathrm{SEM}$ ) and ABC transporter ( $26.7 \pm 1.9 \mathrm{SEM}$ ) genes. ABC, ATP-binding cassette; kbp, kilobase pair; SEM, standard error of the mean; SLC, solute carrier; SNV, single-nucleotide variant; UGT, UDP-glucuronosyltransferase. 
a

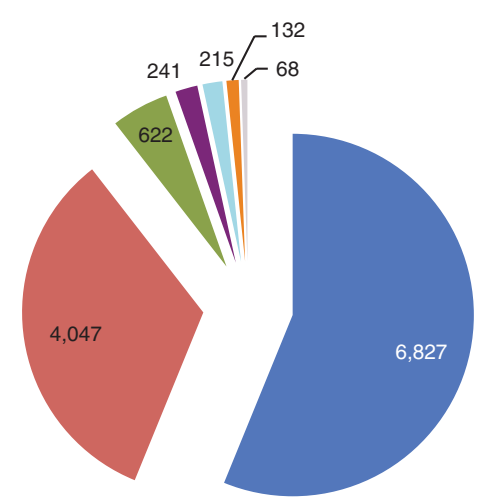

C

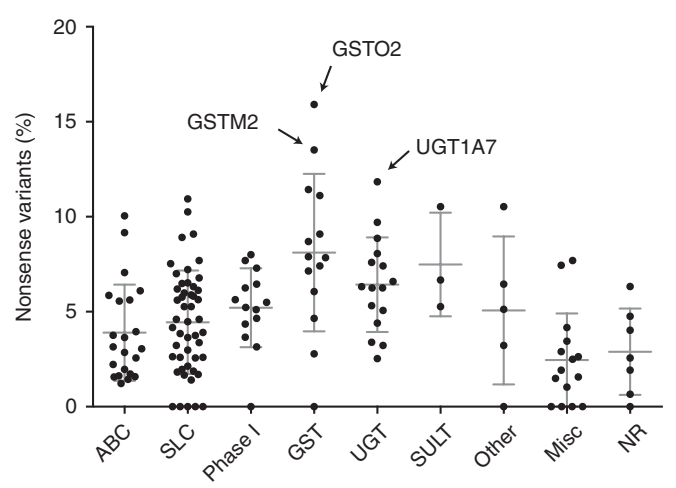

b
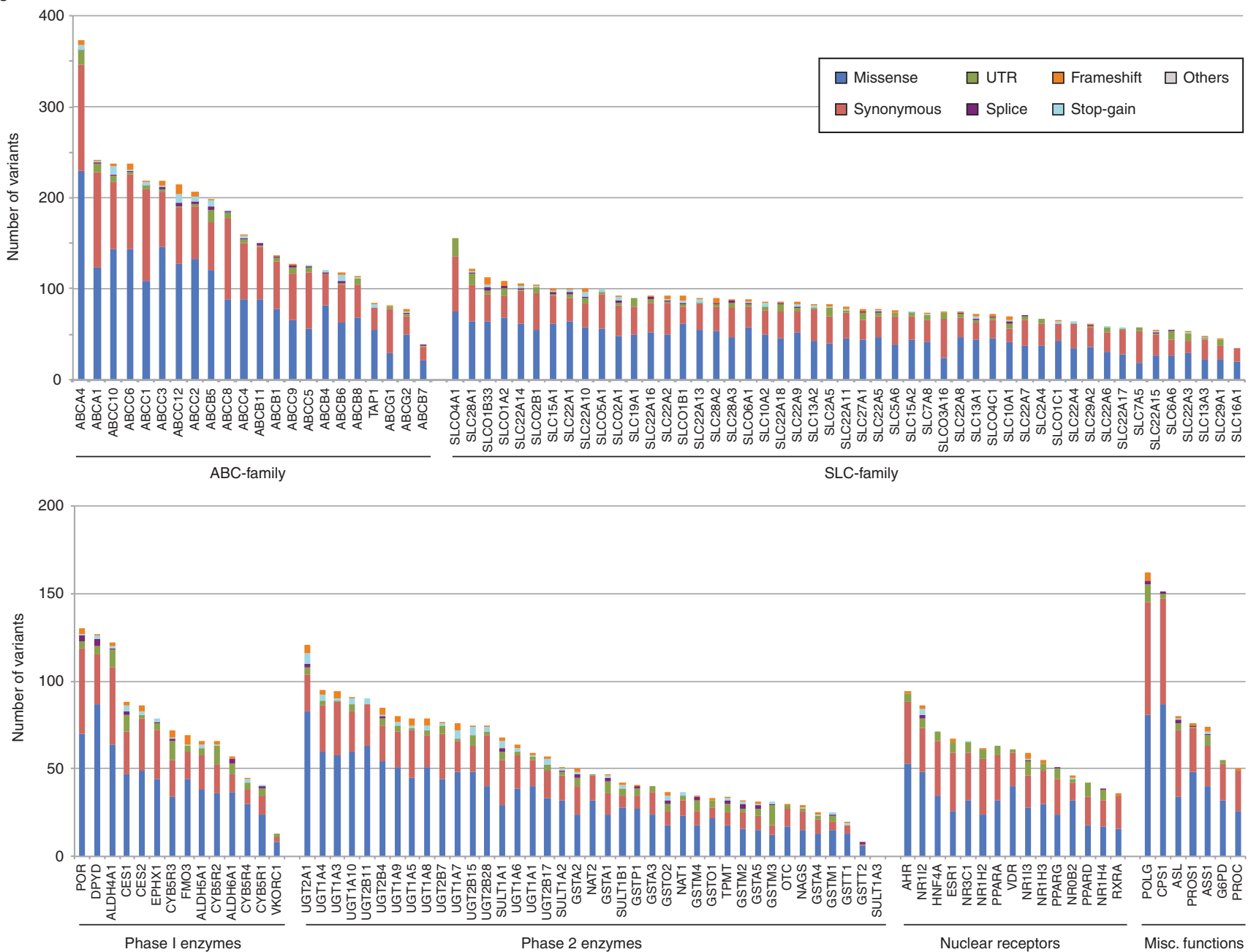

Figure 2 Variant spectra differ substantially between pharmacogenes. (a) Pie chart visualizing the composition of all exonic variants we detected in the integrated data sets. Note that the majority (56.2\%) of variants caused amino acid changes. (b) Overview of the variant class frequencies in all 146 genes that were investigated in this study. Stacked columns are ordered by the total number of variants found in each gene family. For an overview of the number of variants normalized by gene length, see Supplementary Figure S1 online. (c) Scatter plot showing the fraction of nonsense variants (frameshift, splice, or stop-gain variants) for each gene. Overall, the highest fractions of nonsense variants were detected in GSTO2 (15.9\%), GSTM2 (13.5\%), and UGT1A7 (11.8\%).

transporter, and its variants have been linked to Stargardt disease, a common form of autosomal-recessive macular dystrophy. ${ }^{29} A B C C 3$ is highly expressed in liver, and SNVs affect ADR susceptibility to cisplatin. ${ }^{30}$
When taking gene length into account, GSTA2 (59.2 SNVs/ kbp), SLCO4A1 (56.2), SLC22A14 (53.6), and SLC22A1 (53) were most variable, whereas RXRA (6) and SULT1A3 (0) were most conserved (see Supplementary Figure S1 online). 
Variants in GSTA2 are predictive of the outcome of allogenic stem cell transplantations at preparative stages. ${ }^{17}$ SLC22A1 encodes hOCT1, a broad spectrum transporter that impacts PK of the antidiabetic compound metformin, as well as multiple other antiviral and anticancer drugs such as lamivudine and imatinib. ${ }^{31}$

Previous studies estimated that nonsense variants that affect stop-codons, caused frameshifts or occurred in splice sites account for one-third of inherited genetic disorders and are of fundamental importance for human disease. ${ }^{32}$ Hence, we analyzed the contribution of nonsense variants and found that, overall, $4.8 \%$ of variants were such variations, an abundance, which exceeds the value obtained for all GENCODE regions $(1.3 \%) .{ }^{23}$ Relative to the total number of variants detected in each gene family, GSTs and UGTs showed the highest incidences of nonsense variants, with GSTO2, GSTM2, and UGT1A7 having the highest fractions (Figure 2c).

\section{In silico prediction of functional effects of 12,152 SNVs}

Next, we assessed the functional impacts of variants using in silico prediction algorithms. To this end, we used an array of static and machine learning algorithms and evaluated their predictive power on a panel of 24 pharmacogene variants whose functional effects had previously been experimentally and/or clinically validated (see Supplementary Table S3 and Supplementary References online). PolyPhen-2, SIFT, and SNPs 3D classified 17/24 (71\%), 17/24 (71\%), and 13/24 (54\%) missense variants as putatively deleterious, respectively. When integrating all algorithms, 21/24 (88\%) validated functional variants were flagged as potentially damaging. Three functional missense variants in $A B C B 11$ (rs11568357), ABCG2 (rs2231142), and GSTP1 (rs1695) were classified as benign by all algorithms, indicating that their predictive power is still not optimal.

We complemented our functionality assessments with genomic evolutionary rate profiling and Grantham scores. Furthermore, we considered all variants that affected stop codons or resulted in frameshifts or splice defects to be putatively functional. As a result, we classified 6,750 SNVs as putatively functional, with the highest numbers of deleterious mutations being in $A B C A 4$ (236) and $A B C C 10$ (145). Functional variants primarily caused amino acid changes (79.8\%), followed by synonymous (9.9\%) and frameshift (3.6\%) variants (Figure 3a).

We then analyzed the extent of functional polymorphisms across all 146 pharmacogenes (Figure 3b) and the CYP-13 (see Supplementary Figure S2 online). Thus, we calculated the average numbers of functional variants within each gene and determined which fraction of this functional variation was attributed to rare $(\mathrm{MAF}<1 \%)$ and common $(\mathrm{MAF} \geq 1 \%)$ variants. Interestingly, the extent of rare functional variants differed considerably between pharmacogenes from $0.7 \%$ for SLC15A2 to $100 \%$ for, for example, NR1I3 and was not significantly different compared with $C Y P$ genes $(P=0.33$ compared with other phase I enzymes, $P=0.65$ compared with all 146 pharmacogenes).

\section{Genetic variants differ between individuals of European or African descent}

To predict the clinical impact of genetic variants, it is important to consider their distributions and frequencies across different populations. We thus analyzed the variant distributions in populations with European and African ancestry. Interestingly, the majority of variants in pharmacogenes (72\%) were populationspecific (5,100 and 3,977 for Europeans and Africans, respectively, Figure 4a) and, unlike in the CYP supergene family, the proportion of very rare pharmacogene variants was higher in Europeans than in Africans ${ }^{6}$ (Figure $4 \mathrm{~b}$ ).

Each individual of European descent harbored, on average, a total of 100.8 SNVs, whereas the number of variants observed in Africans was 121.4. Highest variation rates in European Americans were observed in UGT2B28 (3.21), ABCC6 (2.92), and $A B C B 5$ (2.31), whereas in Africans $A B C A 1$ (3.8), $A B C A 4$ (3.33), and $A B C C 12$ (3.21) were most variable (Figure 4c). Variants in the ABCC6 gene have been linked to docetaxel and thalidomide response, ${ }^{33}$ whereas SNVs in $A B C A 1$ have been shown to significantly associate with response to several statins ${ }^{34}$ thus indicating potential population-specific effects of statin intake. Some genes showed tremendous differences in their variation rate between European and African Americans; prominent examples include VKORC1 $\left(\mathrm{VR}_{\mathrm{EA}}=0.02, \mathrm{VR}_{\mathrm{AA}}=\right.$ $0.2), G 6 P D\left(\mathrm{VR}_{\mathrm{EA}}=0.12, \mathrm{VR}_{\mathrm{AA}}=0.91\right)$, and $A B C B 1\left(\mathrm{VR}_{\mathrm{EA}}=1.6\right.$, $\mathrm{VR}_{\mathrm{AA}}=0.8$; Figure $4 \mathrm{c}$ inlet).

Combined, our findings indicate that the genetic variability in clinically relevant pharmacogenes differs considerably between European and African Americans, and they pinpoint potential priority genes for population-adjusted genetic profiling strategies.

\section{DISCUSSION}

In the study presented here, we detected a total of 12,152 exonic SNVs in 146 pharmacogenes with clinically important roles, of which the majority were rare (MAF $<1 \%, 92.9 \%$ ) or very rare (MAF $<0.1 \%, 82.7 \%)$. This emphasizes the need to expand the scope from candidate gene analyses in phenotypically different individuals and classical genome-wide association studies that only consider common genetic variants to unbiased sequencing-based approaches. Yet, current sequencing-based technologies like whole-genome sequencing and whole-exome sequencing can face limitations in highly repetitive genomic loci and highly paralogous gene families. To this end, we systematically assessed the fraction of sequence that poses problems for reliable variant calls and only found a weak correlation to the number of SNVs found $\left(R^{2}=0.033, P=0.03\right)$. Thus, short-read sequencing seems suitable for analyzing genetic variation in the pharmacogenes selected here. Nevertheless, it is recommended to validate candidate variants of interest by, for example, classical Sanger sequencing.

Using an array of static and machine learning approaches that consider amino acid physiochemical properties, solvent accessibility as well as structural data, 6,750 SNVs (55.5\%) were flagged as putatively functional. Interestingly, when analyzing 

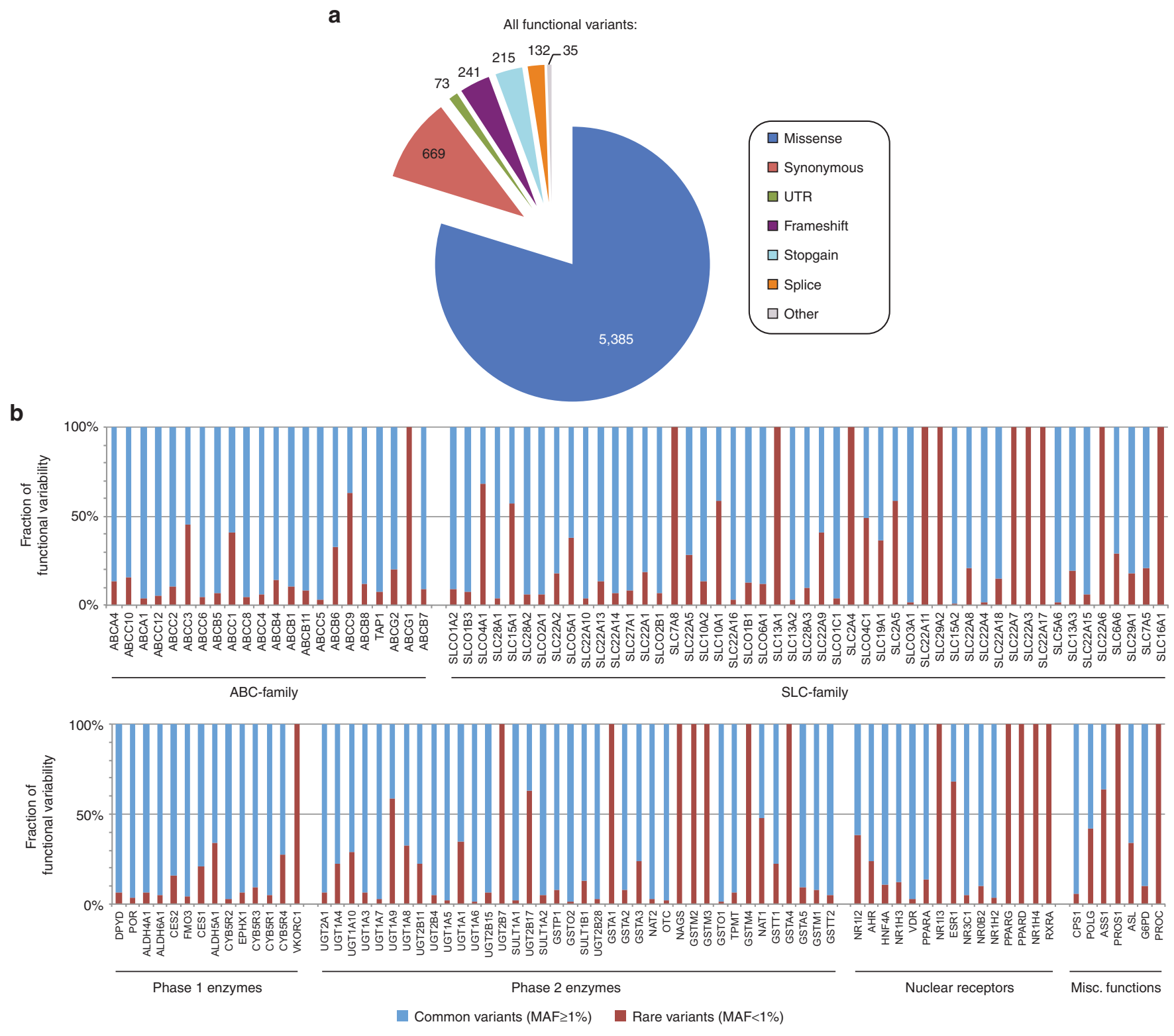

Figure 3 The fraction of functional variability that is attributed to rare and common variants differs strongly between genes. (a) Most putatively functional variants caused amino acid changes (79.8\%) or occurred in conserved synonymous bases (9.9\%). (b) The probability that a functional variant in a pharmacogene is common (MAF $\geq 1 \%$, shown in blue) or rare (MAF $<1 \%$, red) was calculated for all 146 genes in our panel. Importantly, the fraction of functional variation that is allotted to rare variants differs considerably between genes. Genes within each class are ordered by the total number of functional variants found.

the contribution of rare variants to putative gene functionality, we found that the extent to which rare variants contribute to functional effects differed significantly between genes. On the basis of these data, we approximated to what extent rare variants contribute to overall interindividual variability in drug response. A previous study showed that rare variants in SLCO1B1 explained $17.8 \%$ of the variability in clinical efficacy and toxicity of methotrexate treatment and had larger effect sizes than common variants, thus highlighting the importance of rare variants for pharmacogenetic phenotypes. ${ }^{35}$ Consistent with these findings, we observed that $12.7 \%$ of functional variation in SLCO1B1 was attributed to rare variants in our data set. We then extrapolated these values to the overall contributions of rare variants in the pharmacogene panel analyzed. Importantly, $31.9 \%$ of the inherited interindividual variation in these genes was found to be attributed to rare variants, which is approximately 2.5 -fold higher than the value observed in SLCO1B1. We thus concluded that the contribution of rare variants to phenotypic consequences is clinically relevant, and we estimated that approximately $30-40 \%$ of the overall functional variability in pharmacogenes is caused by rare variants with $\mathrm{MAF}<1 \%$. This value is similar to the $28.7 \%$ of functionality that is putatively explained by rare variants in the CYP-13. Yet, it has to be noted that these estimations are based on the contribution of rare variants to clinical treatment outcome of a single drug and gene and require further experimental validations. 
a
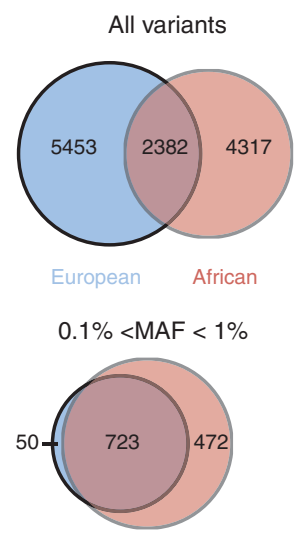

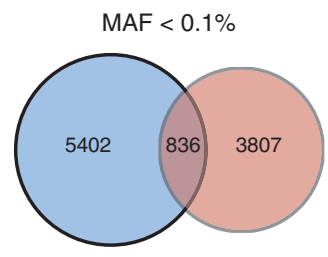

$\mathrm{MAF}>1 \%$

c
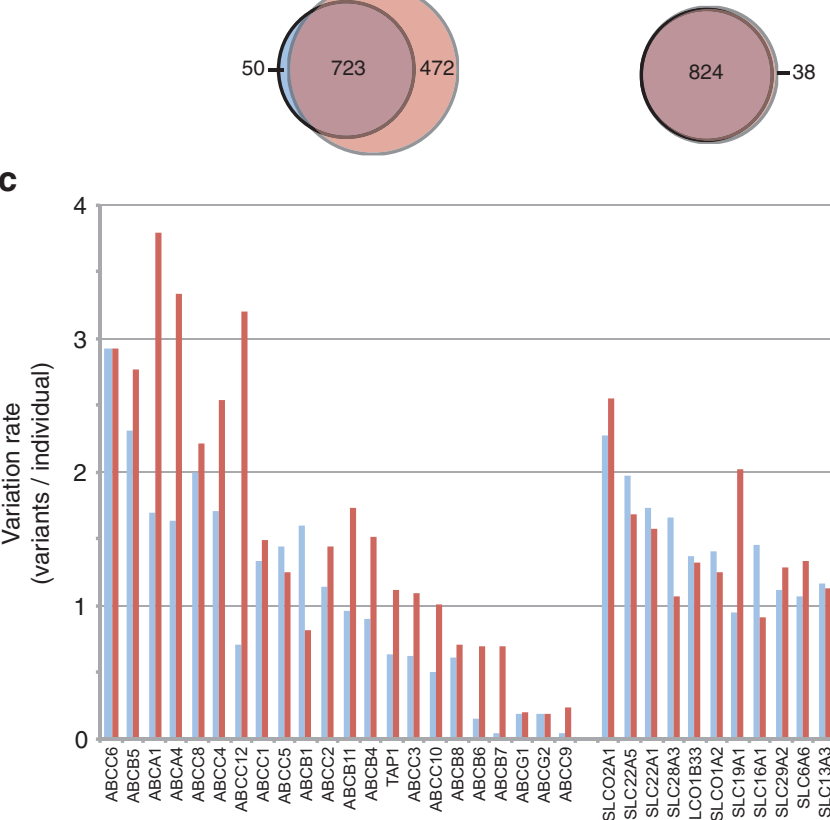

b

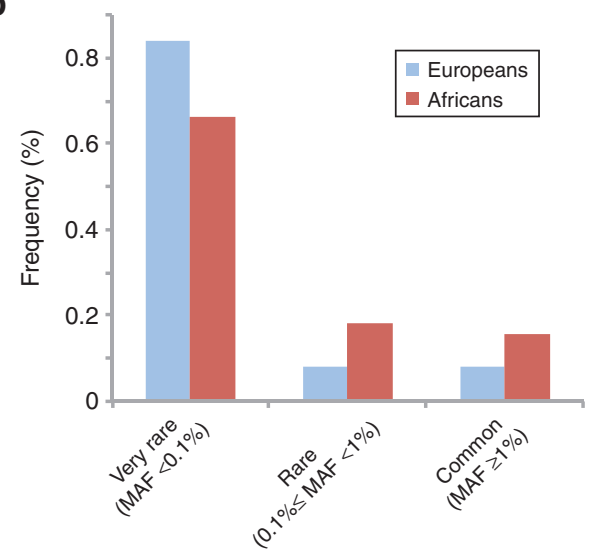

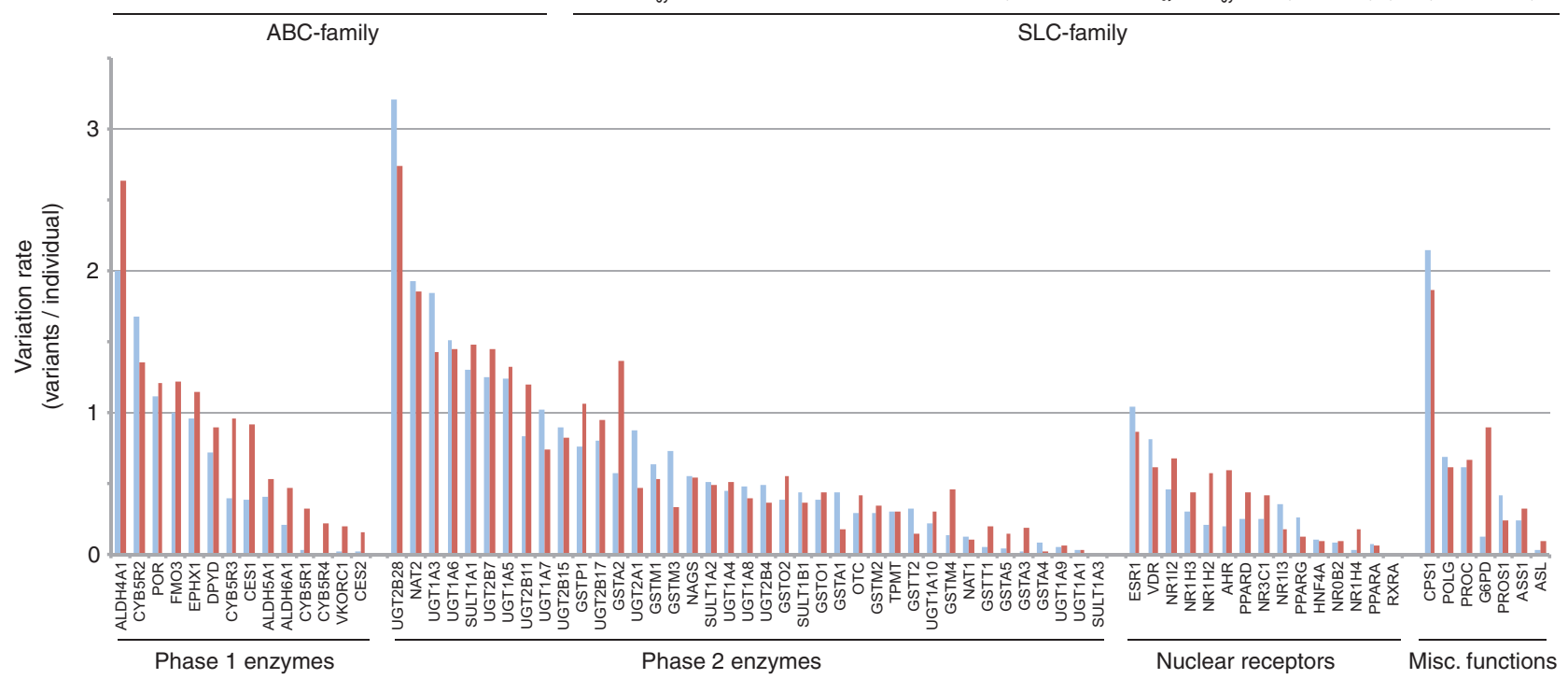

Figure 4 Variation profiles differ markedly between European and African subpopulations. (a) Venn diagrams showing the numbers of shared and unique variants across populations. Overall, $80.4 \%$ of variants were exclusively detected in either European (blue) or African (red) populations. Although no common variants (MAF $>1 \%$ ) were restricted to Europeans, 38 common variants were detected only in Africans. (b) Column plot showing the relative frequencies of very rare (MAF $<0.1 \%)$, rare $(0.1 \% \leq \mathrm{MAF}<1 \%)$, and common (MAF $\geq 1 \%$ ) variants. The fraction of very rare variants was higher in individuals of European ancestry compared with Africans. (c) The variation rates (see the Materials and Methods section for details on the calculation), defined as the average numbers of variants observed per individual, were calculated for each of the 146 pharmacogenes in both European (blue) and African (red) populations. The genes that differed between the populations by more than 0.5 variants/individual are shown in the inlet. Bars are colored red when higher variability was observed in Africans and in blue when higher variability was found in Europeans. 


\section{ORIGINAL RESEARCH ARTICLE}

Furthermore, we analyzed the genetic variability in pharmacogenes in American individuals of European and African descent and found that the genetic diversity differed substantially between individuals of African and European descent, consistent with previous studies. ${ }^{6,28}$ Genetic variability was overall higher in Africans because of complex population dynamics, substantial variability in environmental factors, and differential exposure to infectious disease. ${ }^{36}$ Yet, the genetic composition of indigenous European, and especially African, populations can differ tremendously from the genetic inventory observed in European and African Americans, and thus extrapolation of these findings needs to be performed cautiously.

Although most genes showed increased variability in Africans, there were notable exceptions such as $A B C B 1$, which encodes the multidrug resistance transporter P-Gp, and SLC22A3, encoding a transporter for the antidiabetic drug metformin, ${ }^{37}$ that were more variable in Europeans. The genetic differences on a population level in pharmacogenes provided here can serve as a reference for the design of randomized controlled trials, because the participants of such trials are generally not representative of the target population and might lack external validity. ${ }^{38}$ It is therefore reasonable to assume that extrapolation of trial results becomes more problematic with increasing genetic variability in loci of importance for trial compound PK and PD, and thus optimal treatment regimens and susceptibility to ADRs can differ between populations.

To improve pharmacogenetic utility and human health, the future key task will be to identify variants that are clinically relevant and that provide cost-effective targets for pharmacogenetic testing. While sequencing costs continue to decline, the costs of analysis, annotation, and data management decrease (if at all) to a much lesser extent, thus shifting the bottleneck away from technical considerations toward downstream analyses. ${ }^{39}$ Importantly, current prediction algorithms are trained on data sets linked to disease. Yet, accurate functionality predictions of variants that do not cause phenotypes per se but only modulate PK and PD and result in phenotypes only in certain contexts might follow different rules. Thus, such predictions would benefit from approaches that parameterize the prediction model using sets of validated variants with pharmacogenetic impacts. Hence, there is a need for larger panels of experimentally validated genetic pharmacogene variants that, in turn, will facilitate more accurate in silico predictions of uncharacterized variants. Furthermore, current functionality prediction algorithms generally do not consider haplotype phases because these are usually poorly resolved by short-read sequencing. For instance, the variant rs12248560 is responsible for the increased activity of the CYP2C19*17 allele. Yet, when combined with rs 28399504 , the resulting haplotype (CYP2C19*4B) results in a loss-of-function allele, demonstrating that the effect of SNVs can be very different, depending on whether they occur isolated or in combination with other variants. As a consequence, accurately phased data can serve as a better pharmacogenetic marker than individual SNVs and can improve predictability of drug response. ${ }^{40}$
Although the extent of genetic variations in transporters, phase I and II enzymes, and nuclear receptors is considerable, current pharmacogenetic tests only probe a minute selection of variants and thus do not capture the true interindividual variability, resulting in potentially flawed phenotype predictions and avoidable lack of treatment efficacy. Based on our variant quantifications, we estimate that approximately $30-40 \%$ of functional variability can be allotted to rare variants that are not commonly considered in genetic tests. The clinical implementation of strategies that accommodate for this rare genetic variability is, however, not easy because precision medicine initiatives in which drug treatment is adjusted to comply with the genetic makeup of the specific patient will be complicated by the large extent of rare variants. In principle, two levels of genetic analyses must be considered: (i) most pharmacogenomic drug labels relate to variations in one or a few specific genes and the data presented here indicate that complete gene sequencing coupled to reliable functionality assessment of the variants found would be required and (ii) the functional impact of the extensive genetic variation in all pharmacogenes with relevance for a specific drug treatment must be considered. In contrast to the current pharmacogenomic labels for which the findings of many clinical studies are often available to inform decisions regarding drug choice and dose, it would require major effort to directly link interindividual differences in drug response to the presence of rare pharmacogenetic variations.

In conclusion, the results provided here shed light on the genetic diversity of pharmacogenes and aim to incentivize the use of comprehensive sequencing-based approaches for pharmacokinetic and pharmacodynamic phenotyping. Furthermore, the described variants of interest can serve as a basis for experimental validations and the development of future genotyping platforms.

\section{ACKNOWLEDGMENTS}

V.M.L. was supported by a Marie Curie IEF fellowship for career development in the context of the European FP7 framework program. The project was supported by a grant (5949) from the Swedish Research Council.

\section{DISCLOSURE}

The authors declare no conflict of interest.

\section{SUPPLEMENTARY INFORMATION}

Supplementary material is linked to the online version of the paper at http://www.nature.com/gim.

\section{REFERENCES}

1. Ingelman-Sundberg M. Pharmacogenomic biomarkers for prediction of severe adverse drug reactions. N Engl J Med 2008;358:637-639.

2. Tufts Center for the Study of Drug Development. How the Tufts Center for the Study of Drug Development pegged the cost of a new drug at $\$ 2.6$ billion. 18 November2014. http://csdd.tufts.edu/files/uploads/cost_study_backgrounder. pdf.

3. Frueh FW, Amur S, Mummaneni P, et al. Pharmacogenomic biomarker information in drug labels approved by the United States food and drug administration: prevalence of related drug use. Pharmacotherapy 2008;28:992-998. 
4. Ehmann F, Caneva L, Prasad K, et al. Pharmacogenomic information in drug labels: European Medicines Agency perspective. Pharmacogenomics J 2015;15:201-210.

5. Moaddeb J, Haga SB. Pharmacogenetic testing: current evidence of clinical utility. Ther Adv Drug Saf 2013;4:155-169.

6. Fujikura K, Ingelman-Sundberg M, Lauschke VM. Genetic variation in the human cytochrome P450 supergene family. Pharmacogenet Genomics 2015;25:584-594.

7. Pirmohamed M. Personalized pharmacogenomics: predicting efficacy and adverse drug reactions. Annu Rev Genomics Hum Genet 2014;15:349-370.

8. Franke RM, Gardner ER, Sparreboom A. Pharmacogenetics of drug transporters. Curr Pharm Des 2010;16:220-230.

9. Roundhill EA, Fletcher J, Haber M, Norris MD. Clinical relevance of multidrugresistance-proteins (MRPs) for anticancer drug resistance and prognosis In: Efferth T (ed). Resistance to Targeted ABC Transporters in Cancer. Springer: Heidelberg, Germany, 2015;27-52.

10. Oshiro C, Mangravite L, Klein T, Altman R. PharmGKB very important pharmacogene: SLCO1B1. Pharmacogenet Genomics 2010;20:211-216.

11. Niemi M, Backman JT, Kajosaari LI, et al. Polymorphic organic anion transporting polypeptide 1B1 is a major determinant of repaglinide pharmacokinetics. Clin Pharmacol Ther 2005;77:468-478.

12. The SEARCH Collaborative Group. SLCO1B1 variants and statin-induced myopathy—a genomewide study. N Engl J Med 2008;359:789-799.

13. Kalliokoski A, Neuvonen M, Neuvonen PJ, Niemi M. The effect of SLCO1B1 polymorphism on repaglinide pharmacokinetics persists over a wide dose range. Br J Clin Pharmacol 2008;66:818-825.

14. Guillemette $C$. Pharmacogenomics of human UDP-glucuronosyltransferase enzymes. Pharmacogenomics J 2003;3:136-158.

15. Iyer $L$, Das $S$, Janisch $L$, et al. UGT1A $1 * 28$ polymorphism as a determinant of irinotecan disposition and toxicity. Pharmacogenomics J 2002:2:43-47.

16. Hayes JD, Flanagan JU, Jowsey IR. Glutathione transferases. Annu Rev Pharmacol Toxicol 2005:45:51-88.

17. Bonifazi F, Storci G, Bandini G, et al. Glutathione transferase-A2 S112T polymorphism predicts survival, transplant-related mortality, busulfan and bilirubin blood levels after allogeneic stem cell transplantation. Haematologica 2014;99:172-179.

18. James MO, Ambadapadi S. Interactions of cytosolic sulfotransferases with xenobiotics. Drug Metab Rev 2013;45:401-414.

19. Cook I, Wang T, Falany CN, Leyh TS. High accuracy in silico sulfotransferase models. J Biol Chem 2013;288:34494-34501.

20. Edavana VK, Dhakal IB, Yu X, Williams S, Kadlubar S. Sulfation of 4-hydroxy toremifene: individual variability, isoform specificity, and contribution to toremifene pharmacogenomics. Drug Metab Dispos 2012;40:1210-1215.

21. Omiecinski CJ, Vanden Heuvel JP, Perdew GH, Peters JM. Xenobiotic metabolism, disposition, and regulation by receptors: from biochemical phenomenon to predictors of major toxicities. Toxico/ Sci 2011;120(suppl 1):S49-S75.

22. Tennessen JA, Bigham AW, O'Connor TD, et al.; Broad GO; Seattle GO; NHLBI Exome Sequencing Project. Evolution and functional impact of rare coding variation from deep sequencing of human exomes. Science 2012;337:64-69.
23. 1000 Genomes Project Consortium. An integrated map of genetic variation from 1,092 human genomes. Nature 2012;491:56-65.

24. Drögemöller BI, Wright GE, Niehaus DJ, Emsley R, Warnich L. Next-generation sequencing of pharmacogenes: a critical analysis focusing on schizophrenia treatment. Pharmacogenet Genomics 2013;23:666-674.

25. Yue P, Melamud E, Moult J. SNPs3D: candidate gene and SNP selection for association studies. BMC Bioinformatics 2006;7:166.

26. Coulon S, Gaillard PH, Chahwan C, McDonald WH, Yates JR 3rd, Russell P. Slx1SIX4 are subunits of a structure-specific endonuclease that maintains ribosomal DNA in fission yeast. Mol Bio/ Cell 2004;15:71-80.

27. Tukey RH, Strassburg CP. Human UDP-glucuronosyltransferases: metabolism, expression, and disease. Annu Rev Pharmacol Toxicol 2000;40:581-616.

28. Nelson MR, Wegmann D, Ehm MG, et al. An abundance of rare functional variants in 202 drug target genes sequenced in 14,002 people. Science 2012;337:100-104.

29. Allikmets R, Dean M. Bringing age-related macular degeneration into focus. Nat Genet 2008;40:820-821.

30. Pussegoda K, Ross CJ, Visscher H, et al.; CPNDS Consortium. Replication of TPMT and ABCC 3 genetic variants highly associated with cisplatin-induced hearing loss in children. Clin Pharmacol Ther 2013;94:243-251.

31. Arimany-Nardi C, Koepsell H, Pastor-Anglada M. Role of SLC22A1 polymorphic variants in drug disposition, therapeutic responses, and drug-drug interactions. Pharmacogenomics J 2015;15:473-487.

32. Frischmeyer PA, Dietz HC. Nonsense-mediated mRNA decay in health and disease. Hum Mol Genet 1999;8:1893-1900.

33. Deeken JF, Cormier T, Price DK, et al. A pharmacogenetic study of docetaxel and thalidomide in patients with castration-resistant prostate cancer using the DMET genotyping platform. Pharmacogenomics J 2010;10: 191-199.

34. Voora D, Shah SH, Reed CR, et al. Pharmacogenetic predictors of statinmediated low-density lipoprotein cholesterol reduction and dose response. Circ Cardiovasc Genet 2008;1:100-106.

35. Ramsey LB, Bruun GH, Yang W, et al. Rare versus common variants in pharmacogenetics: SLCO1B1 variation and methotrexate disposition. Genome Res 2012;22:1-8

36. Campbell MC, Tishkoff SA. African genetic diversity: implications for human demographic history, modern human origins, and complex disease mapping. Annu Rev Genomics Hum Genet 2008;9:403-433.

37. Nies AT, Koepsell H, Winter $\mathrm{S}$, et al. Expression of organic cation transporters OCT1 (SLC22A1) and OCT3 (SLC22A3) is affected by genetic factors and cholestasis in human liver. Hepatology 2009;50:1227-1240.

38. Hartman, E., Grieve, R., Ramsahai, R. \& Sekhon, J. S. From sample average treatment effect to population average treatment effect on the treated: combining experimental with observational studies to estimate population treatment effects. J R Stat Soc Ser A 2015;178:757-778.

39. Sboner A, Mu XJ, Greenbaum D, Auerbach RK, Gerstein MB. The real cost of sequencing: higher than you think! Genome Biol 2011;12:125.

40. Snyder MW, Adey A, Kitzman JO, Shendure J. Haplotype-resolved genome sequencing: experimental methods and applications. Nat Rev Genet 2015; 16:344-358. 